\title{
Analysis of Drawbacks and Constraints of Classification Algorithms for Three-Phase Voltage Dips
}

\author{
Jorge L. Strack ${ }^{1,2,3}$, Ignacio Carugati ${ }^{1,2,3}$, Carlos M. Orallo ${ }^{1,2,3}$, Patricio G. Donato ${ }^{1,2,3}$, \\ Sebastián Maestri ${ }^{1,2,3}$, Daniel Carrica ${ }^{1,2,3}$
}

\begin{abstract}
Voltage events are one of the most common and harmful disturbances of power electric systems. Voltage dips, swells and interruptions are included under this heading. Given the economic cost that these disturbances represent for electrical power transmission and distribution companies and the industry, it becomes imperative to detect and classify them properly. Several classification criteria and algorithms have been proposed in the literature as analysis tools to differentiate voltage events by their characteristics and, if possible, to determine their causes and consequences. Even though some of these approaches make a correct classification of the voltage events, there are certain operation conditions that are common in real electrical grids, in which the classification criteria, and their corresponding algorithms, make a wrong classification. These particular conditions, together with the lack of a fair comparison in a common scenario, have not been addressed in the specific field literature. This work explores in detail all these aspects by evaluating the symmetrical components criterion and ABC classification criterion, and rigorously analyzes three specific algorithms: the Symmetrical Components Algorithm, the Six Phases Algorithm and the Space Vector Algorithm. Drawbacks arise from both classification criteria and algorithms. The causes of the classification errors are described and discussed in detail in order to better understand the problem, and evidence the constraints of these classification methods. Copyright (C) 2018 Praise Worthy Prize S.r.l. - All rights reserved.
\end{abstract}

Keywords: Voltage Dips, Voltage Event Classification, Voltage Interruptions, Voltage Swells

\begin{tabular}{|c|c|}
\hline & re \\
\hline$\alpha=\arg \left(Z_{f} / Z_{s}\right)$ & Phase angle between impedances \\
\hline$\beta$ & Argument of the $V_{\beta}$ phasor \\
\hline$V_{a 1}, V_{b 1}, V_{c 1}$ & Phasors of the three-phase voltages \\
\hline$\varphi_{+1}$ & $\begin{array}{l}\text { Phase angle of the positive sequence } \\
\text { component of the fundamental voltage }\end{array}$ \\
\hline$\varphi_{-1}$ & $\begin{array}{l}\text { Phase angle of the negative sequence } \\
\text { component of the fundamental voltage }\end{array}$ \\
\hline$\varphi_{\text {inc }}$ & $\begin{array}{l}\text { angle of the ellipse formed } \\
y \text { of the space vector }\end{array}$ \\
\hline$a$ & $\begin{array}{l}\text { Unit phasor whose phase angle is } \\
120^{\circ}\end{array}$ \\
\hline$A, B, C, \ldots, I^{* *}$ & $\begin{array}{l}\text { Three-phase voltage dips of the } A B C \\
\text { classification }\end{array}$ \\
\hline$b$ & Unit phasor whose phase angle is $60^{\circ}$ \\
\hline$D_{a}, D_{b}, D_{c}$ & $\begin{array}{l}\text { Single-phase voltage dips of the } C S \\
\text { classification }\end{array}$ \\
\hline$C_{a}, C_{b}, C_{c}$ & $\begin{array}{l}\text { Two-phase voltage dips of the } C S \\
\text { classification }\end{array}$ \\
\hline$D_{y n}$ & Delta-star connection with neutral line \\
\hline$F$ & Complex factor, so-called PN factor \\
\hline$h=Z_{F} /\left(Z_{f}+Z_{s}\right)$ & $\begin{array}{l}\text { Reduction factor of the retained } \\
\text { voltage with respect to the pre-fault } \\
\text { voltage }\end{array}$ \\
\hline & Normal state of the network \\
\hline
\end{tabular}

p.u.

PCC

$P N$

$P Q M$

$R$

$R_{f}$

$R_{s}$

$r_{m a}$

$r_{m e}$

$R M S$

$S C$

$S C A$

$S I=r_{m e} / r_{m a}$

$S P A$

SVA

$T$

$T_{F}$

TV

V

$V^{*}$

$V_{\beta}$
Per unit of the nominal or sliding reference voltage

Point of common coupling

Positive negative factor

Power quality monitor

Resistance

Resistance of $Z_{f}$

Resistance of $Z_{s}$

Major axis of the ellipse formed by trajectory of the space vector

Minor axis of the ellipse formed by trajectory of the space vector

Root mean square

Symmetrical components

Symmetrical components algorithm

Shape index

Six-phase algorithm

Space vector algorithm

Main parameter of the $S C A$

New parameter of SCA function of $F$

New parameter of $S C A$ function of $V$

Characteristic voltage

Voltage on the PCC during the fault, so-called retained voltage

Phasor whose argument $\beta$ allows to estimates the $T$ parameter 


$\begin{array}{ll}v_{a}(t), v_{b}(t), v_{c}(t) & \begin{array}{l}\text { Three phase voltages in the time } \\ \text { domain }\end{array} \\ V_{e}(t) & \begin{array}{l}\text { Space vector in the time domain } \\ \text { Source voltage }\end{array} \\ V_{S} & \begin{array}{l}\text { Positive sequence component of the } \\ \text { fundamental voltage }\end{array} \\ V_{+1} & \text { Negative sequence component of the } \\ V_{-1} & \text { fundamental voltage } \\ & \text { Zero sequence component of the } \\ V_{01} & \text { fundamental voltage } \\ & \text { Reactance } \\ X & \text { Reactance of } Z_{f} \\ X_{f} & \text { Reactance of } Z s \\ X_{S} & \text { Impedance between } P C C \text { and the fault } \\ Z_{f} & \text { point } \\ & \text { Impedance between PCC and the } \\ Z_{s} & \text { Source } \\ Z_{f+} & \text { Positive sequence component of } Z_{f} \\ Z_{f-} & \text { Negative sequence component of } Z_{f} \\ Z_{f 0} & \text { Zero sequence component of } Z_{f} \\ Z_{s+} & \text { Positive sequence component of } Z s \\ Z_{s-} & \text { Negative sequence component of } Z s \\ Z_{s 0} & \text { Zero sequence component of } Z s\end{array}$

\section{Introduction}

In the Power Quality field, voltage disturbances are defined as deviations of the actual voltage signal from its ideal counterpart, where the ideal voltage is a sinusoidal waveform with constant amplitude and frequency parameters equal to the nominal ones [1]. One of the most harmful disturbances are events, which are characterized by a perfectly defined start and end times, such as dips, swells and interruptions. According to IEEE 1159-2009 Standard [2], voltage dips are defined as a reduction of the RMS (Root Mean Square) voltage value from 0.1 up to 0.9p.u. (per unit of the nominal or sliding reference voltage) in any of the three phases, with a duration from half-cycle up to one minute. This standard also defines a swell as the increment in the RMS value of the voltage above 1.1 p.u. in any of the three phases with a duration from half-cycle up to one minute; and a voltage interruption as a decrease of the RMS value of the voltage below 0.1 p.u. during less than a minute in any of the three-phases. Even though voltage dips are not harmless as interruptions or swells, they occur more often. Then, the damage produced by the voltage reduction, mainly given by short-circuits, motor starting and transformer energizing, can be higher [3]. As a consequence, their detection and classification have become a key issue for power systems operation, since knowledge about these events allows to determine the type of fault [4], and the source of disturbance and its location [5], among others, and to take corrective actions by clearing the fault, mitigating its effects [6] or avoiding repetition. The detection and classification of a voltage event can be automatically performed, by adopting a suitable classification criterion and implementing the corresponding algorithm in a digital signal processor.
The classification criteria allow determining the type of voltage dip, measuring its severity and providing information about the possible causes. Among the most popular classification criteria are the symmetrical components (SC) and the ABC criteria [7]. SC criterion is more general, since it classifies unbalanced dips as single, double or three-phase dips. Then, the dips produced in an actual network mainly fall within either group. On the other hand, the $\mathrm{ABC}$ criterion is more descriptive and includes a wide variety of events, based on more specific morphological features. For this reason, the $\mathrm{ABC}$ classification criterion is the most widely used, and is adopted in this manuscript. Nonetheless, both criteria are related, as indicated throughout this work.

Once the classification criterion is defined, the distinctive characteristics of the event that permit its identification should be extracted with an algorithm.

Some state-of-the-art works contribute with a theoretical approach about classification algorithms based on heuristic tools, such as fuzzy logics, neural networks, support vector machines, and genetic algorithms, to name a few [8]-[12]. Even though these methods have shown good performance in computer simulations, few experimental verification is provided in the cited works.

In addition, the implementation of these methods requires some particular skills like, for example, a good selection of the model and training sets, which could render them unattractive for industrial applications in which a known and simple method is most preferred. On the other hand, a widely adopted technique is the use of logical algorithms since they can be easily implemented with "nested if" structures.

This key feature justifies the analysis of the following event classification algorithms: the symmetrical components algorithm (SCA) [13], the six-phase algorithm (SPA) [13] and the space vector algorithm (SVA) [14]. Both SCA and SPA were designed to classify dips according to SC classification criterion, whose performance is described in [13].

Despite the fact that some suggestions for classification of voltage dips using $\mathrm{ABC}$ criterion from $\mathrm{SC}$ criterion and estimation of additional parameters are introduced in [15], the algorithms are not implemented and their performance and drawbacks are not analyzed.

On the other hand, the space vector algorithm is designed for $\mathrm{ABC}$ classification. In this case, the drawbacks of the algorithm and its performance under an actual event scenario is not available in the literature [14], [16]-[20]. As regards the algorithm operation, it is demonstrated in the literature that two of the three algorithms evaluated in this work (SCA and SPA) may fail under certain conditions [13], but no comments are made with respect to the third algorithm (SVA). Despite the fact that errors under particular conditions are reported in some works, they are not fully analyzed. In addition, while a topic such as power quality is widely discussed, the specific literature related to this kind of classification criteria and algorithms remains scarce. 
In this work, the distinctive characteristics of the two criteria and the three classification algorithms are identified, the concepts related to classification criteria and algorithms are unified, and a fair comparison under the same operational conditions is conducted.

Furthermore, the characteristics and errors related to each criterion are thoroughly discussed, in order to evidence the real causes of classification errors. It is demonstrated that misclassifications result from not only the algorithms themselves, but also from the classification criterion on which they are based.

Moreover, the errors introduced when the voltage reference is different from the nominal voltage are analyzed. Finally, some aspects related to the possible improvement of the performance of these algorithms are discussed.

Section II introduces the main classification criteria.

Then, the three algorithms under study are presented in Section III, while Section IV describes the limitations of both the algorithms and classification criteria, together with the causes of such limitations. Based on the concepts previously introduced, the algorithms are evaluated with simulations in Section V. Finally, the issues related to the causes leading to criteria and algorithm misclassification are dealt with in Section VI, after which conclusions are drawn in Section VII.

\section{Classification Criteria of Voltage Dips}

A common way of characterizing the severity of a three-phase voltage dip is to determine the lowest RMS value of the three phases during the event, and the highest time interval in which at least one phase is below the threshold of 0.9p.u. [21]. However, this methodology presents some limitations [22]. It classifies single, double or three-phase voltage dips with the same severity, which makes it difficult to draw conclusions regarding the type and location of the fault and does not provide clear information to analyze how a voltage dip propagates down to the power grid, for instance when passing through a transformer. To overcome these limitations, two classification criteria that provide a suitable characterization of the voltage dip are introduced in the work by Bollen and Zhang [7], known as Symmetrical Components (SC) and $\mathrm{ABC}$.

\section{II.1. Symmetrical Components Classification Criterion}

The Symmetrical Components (SC) classification criterion, based on the analysis of voltage symmetrical components, distinguishes between voltage dips with the main voltage decrease in one phase (type $D$ ) and voltage dips with the main voltage decrease in two phases (type $C$ ), as shown in Figs. 1. Dips type $D$ can be classified into $D a, D b$ or $D c$, where the subscript indicates the most affected phase. On the other hand, dips type $C$ can be classified into $C a, C b$ or $C c$, where the subscript indicates the less affected phase. According to this classification, voltage dips can be represented as a phasor with two complex parameters, $V$ and $F$. Factor $V$, known as characteristic voltage, depends on the impedances between the PCC (Point of Common Coupling), the fault location and the sources (see Eq. (1-A) in Appendix). Then, factor $V$ should coincide with the lowest RMS voltage, which could be a line-to-line voltage affected by the factor $\sqrt{3}$ as shown in Fig. 1(a); or a single voltage, as shown in Fig. 1(b). The complex factor $F$, known as the PN (Positive Negative) factor, has a module that depends on the difference between the positive and negative sequence impedances located between the source and the PCC [7].

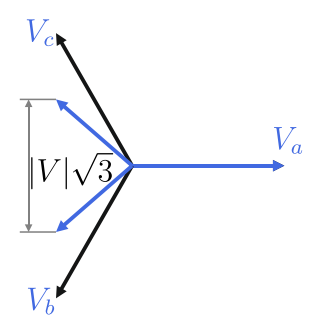

(a)

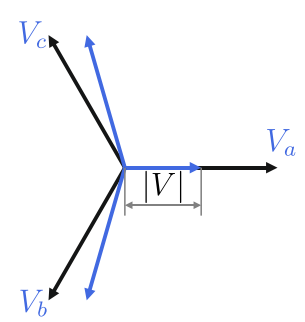

(b)
Figs. 1. Voltage dips of the SC classification: (a) type $\mathrm{Ca}$, and (b) type $\mathrm{Da}$

Each type of dip is defined by a set of equations expressed as a function of $V$ and $F$. For instance, the definition of a type $C a$ is:

$$
\left[\begin{array}{l}
V_{a} \\
V_{b} \\
V_{c}
\end{array}\right]=\left[\begin{array}{c}
F \\
-\frac{1}{2} F-\frac{1}{2} j V \sqrt{3} \\
-\frac{1}{2} F+\frac{1}{2} j V \sqrt{3}
\end{array}\right]
$$

\section{II.2. ABC Classification Criterion}

The ABC classification criterion presented in [1] distinguishes seven types of $\operatorname{dips}(A, \ldots, G)$, and helps to link the event to the originating fault. The method was developed as part of a stochastic prediction of voltage dips. When a statistical analysis of faults is performed, it is possible to calculate how often events occur.

Moreover, the ABC classification allows to synthetize a wide variety of voltage dips; this is particularly useful for testing three-phase equipment that could operate under any of these events when connected to the electrical network. In [23], this criterion has been proposed as a complement to the tests recommended in the IEC 61000-4-11 Standard [24].

Originally, this classification considered a symmetrical voltage $\operatorname{dip}, A$, three types of dips whose main voltage decrease was in one phase, $B, D$ and $F$, and three kinds of dips whose main voltage reduction was in two phases, $C, E$, and $G$. In order to complete the ABC classification, Ignatova [16] proposed three voltage dips combined with swells. The $H$ type, in which the main voltage decrement is in two phases and the remaining 
phase increases its voltage, and the $I^{*}$ and $I^{* *}$ type, whose main voltage decrease is in one phase and the remaining phases experience a voltage increase. The equations that represent the three-phase voltage in $\mathrm{ABC}$ classification are show in the Appendix.

These ones are obtained by combining the contributions of Bollen [1] and Ignatova [16] and expressing the equations as a function of the same variables used in the SC criterion, i.e., the characteristic voltage $\mathrm{V}$ and $\mathrm{PN}$ factor $\mathrm{F}$.

$\mathrm{SC}$ classification is more general than ABC classification, and includes most of the asymmetrical voltage events, which is suitable for a general classification. On the other hand, since ABC classification is more descriptive than $\mathrm{CS}$, it provides more details when a relation between the voltage event and the fault is required. As a consequence, $\mathrm{ABC}$ classification is adopted in the three algorithms analyzed in this work as in [17]-[19].

\section{Algorithms for Voltage Event Classification}

This section presents the operation principles and the main characteristics of the evaluated classification algorithms.

\section{III.1. Symmetrical Components Algorithm (SCA)}

This algorithm, proposed in [13], uses the following expression for events classification:

$$
T=\frac{1}{60} \arg \left\{\frac{V_{-1}}{1-V_{+1}}\right\}
$$

where $V_{-1}, V_{+1}$ are the negative and positive sequence components of the fundamental voltage, respectively.

$T$ is rounded to the nearest integer and can take values between 0 and 5 . From this parameter, it is possible to define the kind of dip in the SC classification, as it can be seen in Table I.

TABLE I

DiP TYPE IN THE SC CLASSIFICATION OF SCA (SECOND ROW) AND OF SPA (THIRD ROW)

\begin{tabular}{ccc}
\hline \hline SC Type & $\mathrm{T}$ & Lowest Voltage \\
\hline$C_{a}$ & 0 & $\mathrm{~V}_{\mathrm{BC} 1}$ \\
$D_{c}$ & 1 & $\mathrm{~V}_{\mathrm{C} 1}$ \\
$C_{b}$ & 2 & $\mathrm{~V}_{\mathrm{CA} 1}$ \\
$D_{a}$ & 3 & $\mathrm{~V}_{\mathrm{A} 1}$ \\
$C_{c}$ & 4 & $\mathrm{~V}_{\mathrm{AB} 1}$ \\
$D_{b}$ & 5 & $\mathrm{~V}_{\mathrm{B} 1}$ \\
\hline \hline
\end{tabular}

As it was mentioned in the previous section, the ABC classification is more suitable to adequately characterize voltage dips.

Hence, in order to obtain the dip type based on the $\mathrm{ABC}$ criterion, it is necessary to obtain three parameters in addition to the $T$ parameter: the characteristic voltage $V$, the PN factor $F$ and the zero-sequence component of the fundamental voltage $V_{01}$.

The parameters $V$ and $F$ are estimated from (3), presented in [15], and $V_{01}$ is obtained from (4):

$$
\begin{gathered}
{\left[\begin{array}{l}
V \\
F
\end{array}\right]=\left[\begin{array}{c}
V_{+1}-b^{6-T} V_{-1} \\
V_{+1}+b^{6-T} V_{-1}
\end{array}\right]} \\
V_{01}=\frac{\left|\dot{V}_{a 1}+\dot{V}_{b 1}+\dot{V}_{c 1}\right|}{3}
\end{gathered}
$$

where $b=1 / 2+j \sqrt{3} / 2$ is a $60^{\circ}$ phase shift and $V_{a 1}, V_{b 1}$ and $V_{c 1}$ are the phasors of the three phases.

Finally, using parameter $T$ and values $V_{01}, F$ and $V$ in Table II, the dip type in ABC classification and the affected phases are obtained.

\section{III.2. Six-Phase Algorithm (SPA)}

This algorithm uses the fundamental RMS single voltages (5) and line-to-line voltages (6), removing the zero-sequence fundamental voltage [13] to classify voltage dips:

$$
\begin{gathered}
{\left[\begin{array}{l}
V_{A 1} \\
V_{B 1} \\
V_{C 1}
\end{array}\right]=\left[\begin{array}{l}
\left|\dot{V}_{a 1}-\dot{V}_{01}\right| \\
\left|\dot{V}_{b 1}-\dot{V}_{01}\right| \\
\left|\dot{V}_{c 1}-\dot{V}_{01}\right|
\end{array}\right]} \\
{\left[\begin{array}{l}
V_{A B 1} \\
V_{B C 1} \\
V_{C A 1}
\end{array}\right]=\frac{1}{\sqrt{3}}\left[\begin{array}{l}
\left|\dot{V}_{a 1}-\dot{V}_{b 1}\right| \\
\left|\dot{V}_{b 1}-\dot{V}_{c 1}\right| \\
\left|\dot{V}_{c 1}-\dot{V}_{a 1}\right|
\end{array}\right]}
\end{gathered}
$$

Voltage dip, according to SC classification [7], is determined by the lowest RMS voltage, as shown in Table I.

Like in the SCA algorithm, it is interesting to know the type of voltage dip based on ABC classification, as well as the affected phases.

This is achieved by using the lowest RMS voltage in Table II (third row) and the value $V_{01}$, which is calculated with the expression (4), $V$ and $F$.

In this case, it can be approximated as follows:

$$
\left[\begin{array}{l}
|V| \\
|F|
\end{array}\right]=\left[\begin{array}{l}
\min \left\{V_{A 1}, V_{B 1}, V_{C 1}, V_{A B 1}, V_{B C 1}, V_{C A 1}\right\} \\
\max \left\{V_{A 1}, V_{B 1}, V_{C 1}, V_{A B 1}, V_{B C 1}, V_{C A 1}\right\}
\end{array}\right]
$$

\section{III.3. Space Vector Algorithm (SVA)}

In [14], [16]-[17], a technique for detecting, classifying and characterizing voltage events based on the analysis of the space vector trajectory in the complex plane is presented. 
The space vector transformation consists in calculating a phasor (8), whose magnitude and phase angle are representative of the three-phase voltages.

Hence, the amount of information required to represent the three-phase voltages is reduced:

$$
V_{e}(t)=\frac{2}{3}\left[v_{a}(t)+a v_{b}(t)+a^{2} v_{c}(t)\right]
$$

with $a=e^{j 2 \pi / 3}$ a phase-shift of $120^{\circ}$.

In a symmetrical three-phase system, the three voltages have the same magnitude and are rotated $120^{\circ}$.

Hence, the trajectory of the space vector is a circle of unitary radius.

In [17], it is demonstrated that a symmetrical dip describes a circumference with a radius proportional to the dip depth, while an asymmetrical dip describes an ellipse. To distinguish between different kind of dips and swells, the parameters of the ellipse (9) and the zerosequence voltage (4) are used:

$$
\left[\begin{array}{c}
r_{m a} \\
r_{m e} \\
\varphi_{i n c} \\
S I
\end{array}\right]=\left[\begin{array}{c}
\left|V_{+1}\right|+\left|V_{-1}\right| \\
|| V_{+1}|-| V_{-1} \mid \\
\frac{1}{2}\left(\varphi_{+1}+\varphi_{-1}\right) \\
\frac{r_{m e}}{r_{m a}}
\end{array}\right]
$$

where $r_{m a}, r_{m e}, \varphi_{i n c}$ and $S I$ are the major axis, minor axis, inclination angle and shape index, respectively, while $\varphi_{+1}$ and $\varphi_{-1}$ are the phase angle of the positive and negative sequence components of the fundamental voltage, respectively.

This classification criterion is provided in Table II, where it can be noticed that, unlike the previous methods, SVA allows to classify not only the voltage dips but also the voltage swells affecting one phase (type $H$ ) or two phases (type $I^{*}$ and $I^{* *}$ ).

\section{Voltage Event Classification Issues}

The state-of-the-art includes some analysis about the errors in the classification algorithms under certain conditions.

However, the causes of this misclassification are not explained in detail.

This work shows that these errors result not only from the particular architecture of each algorithm, but also from the limitations inherent to the classification criteria.

\section{IV.1. Phase Jumps and Asymmetrical Phase Rotations}

As described in [25]-[27], the electrical network faults affect the voltage magnitude in the PCC, and produce phase jumps and phase rotations.

Phase jumps can be attributed to two causes.
Firstly, the difference in the $X / R$ ratio of the impedances between PCC and the fault, $Z_{f}$, and between PCC and the source, $Z_{s}$, originates phase jumps in the $\mathrm{PCC}$ retained voltage.

An exception occurs when $Z_{f}>>Z_{s}$. Analytical description can be found in Appendix. Secondly, the propagation of unbalanced events through transformers that use asymmetrical connection schemes (for instance, $D_{y n}$ ) can lead to phase jumps in points of the electrical network fed by such transformers, even though the original event does not have such phase jump [26].

Regarding the parameters measured by the classification algorithms, phase jumps produce an argument of $V$ different from zero.

On the other hand, asymmetrical phase rotations originate when the positive and negative sequence impedances between PCC and the source are not equal.

In this case, an asymmetrical phase rotation affects the argument of $F$. This feature does not occur with passive elements like lines and transformers, but it is possible in the proximity of generators and motors.

Notice that the SC criterion considers that the zero sequence voltage component is zero $\left(V_{a 1}+V_{b 1}+V_{c 1}=0\right)$; then, from the three expressions that define the threephase voltage, only two are linearly independent. When this feature is included in the six expressions corresponding to each type of dip (for instance, expression (1) for type $C a$ ), each expression generates a set of two equations with two unknowns, $V$ and $F$.

Consequently, when analytically solved, this system could make that the same three-phase voltages $\left(V_{a 1}, V_{b 1}\right.$, $\left.V_{c 1}\right)$ result from any of the six events, each of them with a particular combination of $V$ and $F$. However, these parameters cannot take any value. In general, $|F| \geq|V|$ and the module of $V$ can take any absolute value in so far as $|V|<0.9$ p.u.. Finally, the phase angle of $F$ or $V$ will be different from zero when phase rotations or jumps occur [15].

In spite of the fact that the amplitude and phase angle of $V$ and $F$ would allow to discard some of the six combinations due to their physical impossibility, the existence of high asymmetrical phase rotations or phase jumps that disturb the three-phase voltages will make it difficult to distinguish one kind of event from the other.

This is a major drawback of the classification criterion in general, regardless of the classification algorithm implemented.

This feature is not analyzed in the literature; what is more, misclassification is associated to the classification algorithms performance and not to the classification criterion.

An example of the above mentioned is illustrated in Figs. 2, where the dashed line represents the ideal threephase electric power, the solid black line two ideal events (type $C_{c}$ (a) and type $D_{a}(\mathrm{~b})$ ), and the solid blue line the three phase voltages that result from including phase jumps and/or asymmetrical phase rotations in these events. 
TABLE II

DETERMINATION OF ABC Dip TYPE AND DisTURBED PHASES FROM THE ALGORITHMS SCA, SPA AND SVA

\begin{tabular}{|c|c|c|c|c|c|c|c|c|c|c|}
\hline \multicolumn{9}{|c|}{ Input Variables } & \multirow{2}{*}{\multicolumn{2}{|c|}{$\begin{array}{c}\text { Output } \\
\text { Variables }\end{array}$}} \\
\hline \multicolumn{4}{|c|}{ SCA and SPA } & \multicolumn{5}{|c|}{ SVA } & & \\
\hline $\mathrm{T}$ & $\begin{array}{l}\text { Lowest } \\
\text { voltage }\end{array}$ & $\begin{array}{l}\mid \text { |V01| } \\
\text { [p.u.] }\end{array}$ & $|\mathrm{F}|$ [p.u.] & SI & $\varphi_{\text {inc }}\left[{ }^{\circ}\right]$ & $\varphi_{01}-\varphi_{+1}\left[{ }^{\circ}\right]$ & $\begin{array}{l}\text { |V01| } \\
\text { [p.u.] }\end{array}$ & $\left|\mathrm{r}_{\mathrm{ma}}\right|$ [p.u.] & Type & Phase \\
\hline 3 & $\mathrm{~V}_{\mathrm{A} 1}$ & & & & $-90 \pm 15$ & - & & & & $a$ \\
\hline 5 & $\mathrm{~V}_{\mathrm{B} 1}$ & $\geq 0.033$ & $>|\mathrm{V}| \& \geq 0.967$ & $<1$ & $-30 \pm 15$ & - & $\geq 0.033$ & $\geq 0.967$ & $B$ & $b$ \\
\hline 1 & $\mathrm{~V}_{\mathrm{C} 1}$ & & & & $30 \pm 15$ & - & & & & $c$ \\
\hline 4 & $\mathrm{~V}_{\mathrm{AB} 1}$ & & & & $-60 \pm 15$ & - & & & & $a, b$ \\
\hline 0 & $\mathrm{~V}_{\mathrm{BC} 1}$ & $<0.033$ & $>|\mathrm{V}| \& \geq 0.967$ & $<1$ & $0 \pm 15$ & - & $<0.033$ & $\geq 0.960$ & $C$ & $b, c$ \\
\hline 2 & $\mathrm{~V}_{\mathrm{CA} 1}$ & & & & $60 \pm 15$ & - & & & & $c, a$ \\
\hline 3 & $\mathrm{~V}_{\mathrm{A} 1}$ & & & & $-90 \pm 15$ & - & & & & $a$ \\
\hline 5 & $\mathrm{~V}_{\mathrm{B} 1}$ & $<0.033$ & $>|\mathrm{V}| \& \geq 0.967$ & $<1$ & $-30 \pm 15$ & - & $<0.033$ & $\geq 0.967$ & $D$ & $b$ \\
\hline 1 & $\mathrm{~V}_{\mathrm{C} 1}$ & & & & $30 \pm 15$ & - & & & & $c$ \\
\hline 4 & $\mathrm{~V}_{\mathrm{AB} 1}$ & & & & $-60 \pm 15$ & - & & & & $a, b$ \\
\hline 0 & $\mathrm{~V}_{\mathrm{BC} 1}$ & $\geq 0.033$ & $>|\mathrm{V}| \&<0.967$ & $<1$ & $0 \pm 15$ & - & $\geq 0.033$ & $<0.967$ & $E$ & $b, c$ \\
\hline 2 & $\mathrm{~V}_{\mathrm{CA} 1}$ & & & & $60 \pm 15$ & - & & & & $c, a$ \\
\hline 3 & $\mathrm{~V}_{\mathrm{A} 1}$ & & & & $-90 \pm 15$ & - & & & & $\bar{a}$ \\
\hline 5 & $\mathrm{~V}_{\mathrm{B} 1}$ & $<0.033$ & $>|\mathrm{V}| \&<0.967$ & $<1$ & $-30 \pm 15$ & - & $<0.033$ & $<0.967$ & $F$ & $b$ \\
\hline 1 & $\mathrm{~V}_{\mathrm{C} 1}$ & & & & $30 \pm 15$ & - & & & & $c$ \\
\hline 4 & $\mathrm{~V}_{\mathrm{AB} 1}$ & & & & $-60 \pm 15$ & - & & & & $a, b$ \\
\hline 0 & $\mathrm{~V}_{\mathrm{BC} 1}$ & $<0.033$ & $>|\mathrm{V}| \&<0.967$ & $<1$ & $0 \pm 15$ & - & $<0.033$ & $<0.960$ & $G$ & $b, c$ \\
\hline 2 & $\mathrm{~V}_{\mathrm{CA} 1}$ & & & & $60 \pm 15$ & - & & & & $c, a$ \\
\hline- & - & $<0.033$ & $\approx|\mathrm{V}| \& \leq 0.9$ & $\approx 1$ & - & - & $<1$ & $\leq 0.9$ & $A$ & $a, b, c$ \\
\hline- & - & $<0.033$ & $\approx|\mathrm{V}| \&>0.9 \&<1.1$ & $\approx 1$ & - & - & $<1$ & $>0.9 \&<1.1$ & $N S$ & - \\
\hline & & & & & & $0 \pm 15$ & & & & $a$ \\
\hline- & - & - & - & $\approx 1$ & - & $60 \pm 15$ & $\geq 0.1$ & - & $H$ & $b$ \\
\hline & & & & & & $-60 \pm 15$ & & & & $c$ \\
\hline & & & & & & $-60 \pm 15$ & & & & $a, b$ \\
\hline- & - & - & - & $\approx 1$ & - & $0 \pm 15$ & $\geq 0.1$ & - & $I^{*}$ & $b, c$ \\
\hline & & & & & & $60 \pm 15$ & & & & $c, a$ \\
\hline & & & & & & $-60 \pm 15$ & & & & $a, b$ \\
\hline - & - & - & - & $\approx 1$ & - & $0 \pm 15$ & $\geq 0.033$ & $\geq 0.967$ & $I^{* *}$ & $b, c$ \\
\hline & & & & & & $60 \pm 15$ & & & & $c, a$ \\
\hline
\end{tabular}

Note 1: type NS corresponds to the normal state of the network.

Note 2: all voltages are expressed in [p.u.] with regards to the nominal or declared grid voltage.

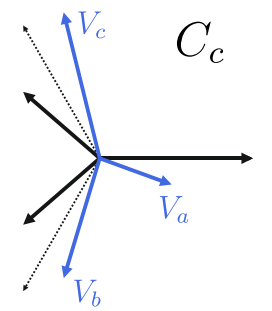

(a)

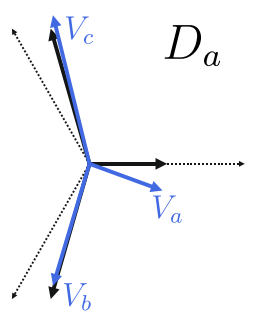

(b)
Figs. 2. Voltage dip type Cc (a) and type Da (b). The ideal three phase electric power is represented in dashed line. In solid black line, two ideal events (type Cc (a) and type Da (b)) are represented and in solid blue line the three-phase voltages that result from including phase jumps and/or asymmetrical phase rotations to these events

Notice that an event is defined as ideal when it is obtained only from variations in the three-phase voltages, i.e., there are no phase jumps or phase rotations associated. In the first case, a $C c$ type event was generated, with $V=0.5 \mathrm{e}^{-\mathrm{j} 20^{\circ}}$ p.u. and $F=1$ p.u. In the second case, a type Da with $V=0.54 \mathrm{e}^{\mathrm{j} 10.95}$ p.u. and $F=0.98 \mathrm{e}^{-\mathrm{j} 16.13^{\circ}}$ p.u. was generated. As it can be observed from this example, there is no way of distinguishing which event occurred, since, in both cases, the disturbed three-phase voltages are the same (blue lines in Figs. 2(a) and (b)), and they were obtained with physically valid combinations of $V$ and $F$.

Therefore, it can be concluded that, regardless of the particular structure of each algorithm, the possibility of a misclassification is always present. Again, this is inherent to the limitations of the classification criterion.

If the same analysis is performed for the $\mathrm{ABC}$ criterion, the conclusion is similar, i.e., the same threephase voltages $\left(V_{a 1}, V_{b 1}\right.$ and $\left.V_{c 1}\right)$ can be obtained from different events with particular combinations of phase jumps and asymmetrical phase rotations.

Since this limitation is related to the way in which the classification criteria was proposed, the classification algorithms present the same limitations. A partial study on the errors of SCA and SPA due to phase jumps and asymmetrical phase rotations is given in [13], which concluded that SCA does not yield errors under a phase jump.

The same applies to SPA with asymmetrical phase rotations. These conditions were also evaluated individually, i.e., events with asymmetrical phase rotations and without phase jumps ( $V=1$ p.u.), and with phase jumps and no symmetrical phase rotations $(F=1$ p.u.). Since the number of independent variables was limited, the tests did not reveal the drawbacks of the criteria with respect to the event classification. Even though these test conditions allow to analyze the effects separately, they are not representative of an actual operational condition.

Finally, a study on SVA errors is not available in the literature [14], [16]-[19]. 


\section{IV.2. Limitations of Event Classifications in the Presence of Symmetrical Phase Rotations and Pre-Fault Voltage Variations Regarding Nominal Magnitude}

The model used to generate faults with phase jumps and asymmetrical rotations in Appendix is based on the pre-fault voltage and line impedance. In this model, the ratio between positive and negative sequences of the grid voltage is explained by some patterns that are used by the algorithms to classify the event.

A condition that is not considered by the above mentioned model is the symmetrical phase rotation of the grid voltages. This condition can be associated to a switchgear operation, the activation of protection devices that connect/disconnect high power loads, or a fault in the reference used in measurement instruments. For instance, there are situations in which the pre-fault current is high due to the connection of a high inductive load. In these cases, the pre-fault voltage is lower than the source voltage because of a voltage drop in the line, and the phase angle is proportional to the sum of the load and line impedance phase. As a consequence, a symmetrical phase rotation is produced when a fault in the line is located near the load, which may be understood as a reference loss of the three-phase voltages with respect to the prefault voltage. One of the effects of this rotation is an event misclassification by SCA and SVA algorithms, since these algorithms use the module and phase of positive and negative sequence voltages as data for the classification process. However, the classification with the SPA algorithm is not affected since it only uses the amplitude of the three-phase voltages and the three line voltages.

Figs. 3 display a particular example in which an event type $D a$ is synthesized, with a retained voltage $V=0.5 \mathrm{e}^{-}$ ${ }^{2} 20^{\circ}$. Fig. 3(a) shows the event generation under ideal conditions, that is to say, without phase jumps and/or symmetrical rotations, using a pre-fault voltage equal to the unity, and measuring the phase angle of the three voltages respect to the pre-fault phase.

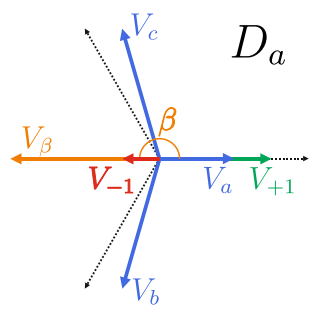

(a)

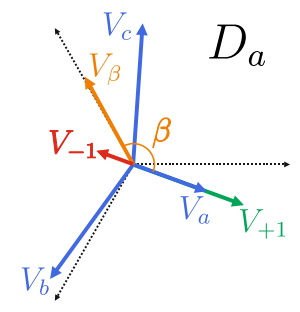

(b)
Figs. 3. Voltage dip type $D a$ with a retained voltage $V=0.5 p . u$. (a) Dip under ideal conditions. (b) Dip with a symmetrical rotation of $-20^{\circ}$ with regard to the prefault voltage. $V_{\beta}$ is the phasor whose argument $\beta$ allows to estimates the value of the parameter $T$, and with it, SCA estimates the event type. $V_{\beta}=V_{-1} /\left(1-V_{+1}\right)$

Under these conditions, the phase angle of the phasor $V_{\beta}=V_{-1} /\left(1-V_{+1}\right)$, so-called $\beta$, is equal to $180^{\circ}$. According to equation (2) and Table I, $T=3$ and the event is correctly classified by the SCA algorithm. In Fig. 3(b), the same voltage event is affected by a symmetrical phase rotation, which produces a difference of $20^{\circ}$ between the three-phase voltages and the corresponding pre-fault voltages. In this case, the positive and negative sequence components are rotated with the same phase angle, but $\beta=119.01^{\circ}$, and so, $T=2$, and considering the criteria of Table I, the event is misclassified as $C_{b}$ rather than as $D_{a}$.

As it was previously mentioned, the loads may produce variations in the module and phase angle of the pre-fault voltage with respect to the nominal voltage. At the same time, the voltage in each point of the electrical network depends on the load as well as on other factors, such as:

- control systems of bulk generators that regulate the voltage level by means of active power control,

- the position of voltage regulation tap of transformers, - capacitor banks for power factor correction,

- etc.

For the reasons exposed, it is normal that a voltage event in any of the grid phases leads to changes in the non-affected phases. This fact may generate classification errors in the algorithms described in Section III, because these are based on static detection thresholds, which are calculated under the assumption that the voltage in the non-affected phases is equal to the nominal voltage.

\section{Performance Evaluation}

This section summarizes the results of the performance evaluation of the three algorithms analyzed in this work. The algorithms were implemented in MATLAB®. For the evaluation, the phasors corresponding to each event type were generated based on the equations in Tables A1 and A2 (see Appendix), and the respective fundamental symmetrical components were estimated. The three algorithms were tested with these signals, comparing the outputs to the known input values. Phase jumps and asymmetrical phase rotations were generated by means of variations in $V$ and $F$ module and phase. Symmetrical phase rotations and prefault voltage variations with respect to the nominal magnitude were generated by means of variations in the module and phase of the reference voltage. Note that the first two disturbances are asymmetric, increasing the unbalance of the three phases. While the last two disturbances are symmetrical, affecting all three phases equally.

\section{V.1. Phase Jumps}

In order to evaluate the performance of the algorithms under the presence of phase jumps, a set of synthetic dips was generated, according to the $\mathrm{ABC}$ classification criterion, with retained voltages from 0 to 1 and phase jumps of the retained voltage ranging from $-90^{\circ}$ to $+90^{\circ}$.

Figs. 4 list the results obtained with $\operatorname{SCA}(a), \operatorname{SPA}(b)$ and SVA(c) algorithms in the case of dips type $C$ and $D$. 


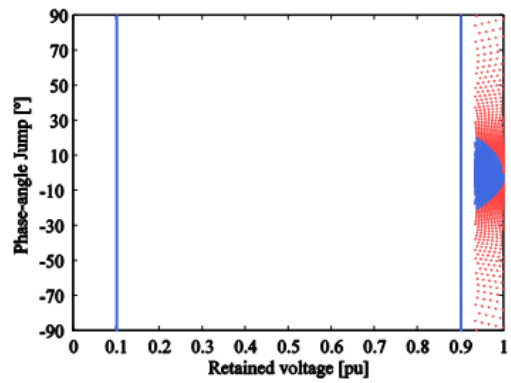

(a)

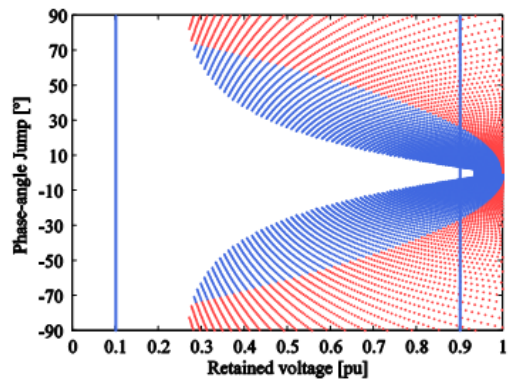

(b)

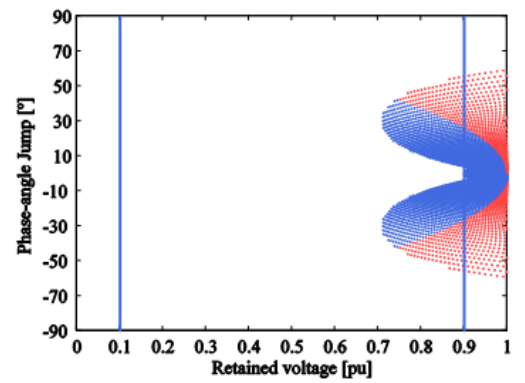

(c)

Figs. 4. Phase jump tests for SCA(a), SPA(b) and SVA(c) algorithms with voltage dips type $C$ and $D$. Blue and red dots indicate that the classification performed by the algorithm was incorrect. Blue dots correspond to combinations of retained voltage and phase jumps that could occur in a radial network, while red dots correspond to combinations that should not be obtained from a radial network. Vertical lines for a retained voltage of 0.1 and 0.9p.u. indicate the limits within which the voltage dips are defined according to IEEE 1159-2009

Blue and red dots indicate the cases in which the algorithm misclassified data. Blue dots correspond to the combinations of retained voltage and phase jumps that are feasible in a radial network, while red dots correspond to combinations that cannot be reproduced in a radial network. The vertical lines for retained voltages of 0.1 and 0.9 p.u. indicate the limits within which voltage dips are defined according to IEEE 1159-2009.

Blue dots are obtained from equation (20) in Appendix, varying the absolute impedance ratio, $\lambda$, in the range of $\left[10^{-4}, 10^{3}\right]$ and the phase angle between impedances, $\alpha$, in the range of $\left[-90^{\circ} ; 90^{\circ}\right]$. The voltage on the PCC during the fault, $V^{*}$, was calculated for each pair of values $(\lambda ; \alpha)$, obtaining the module or retained voltage (represented on the $\mathrm{x}$ axis) and the phase angle (represented on the y axis). The retained voltage $V^{*}$ coincides with the characteristic voltage $V$ in all event types, except for type $\mathrm{B}$, in which the relation is $V=F / 3$ $+2 / 3 V^{*}$.

In Fig. 4(a), it can be observed that SCA is not sensitive to phase jumps and classifies all the voltage events type $C$ and $D$ correctly for any combination of retained voltage and phase jump. This can be explained by the fact that the phase between $V-1$ and $(1-V+1)$ is not affected by the phase jumps.

Additionally, the simulation of phase jumps only affects the phase of $V$, maintaining $F=1$ p.u.. The value of $F$ limits the possible combinations of different voltage events, and so the limitations of the classification criteria described in Section II are not evident. However, this test is useful to understand the individual effects.

Fig. 4(b) shows the results of SPA algorithm. This algorithm is very sensitive to phase jumps, especially when the dips magnitude is higher than 0.3p.u..

SVA algorithm (Fig. 4(c)) has better performance than SPA algorithm, with a phase jump sensitivity only relevant for dips of magnitudes higher than 0.75p.u. (a higher magnitude of retained voltage involves lower voltage reduction). However, this is not a good characteristic, because dips of reduced magnitude (near 0.9p.u.) are the most frequent in the network.

The results obtained when testing other types of events show some variations in relation to Figs. 4, but the three algorithms have similar performance to that of the

\section{classification of dips type $C$ and $D$.}

It is noteworthy that an optimal performance without errors was obtained only for classification of symmetrical events, i.e. A type. This is because symmetrical events have the particular feature that $V=F$.

\section{V.2. Asymmetrical Phase Rotations}

The analysis of the influence of asymmetrical phase rotations on the performance of classification algorithms was conducted by means of two different tests. The first one consisted in the analysis of all the possible combinations of asymmetrical phase rotations and retained voltage for a system in which there are no phase jumps (in other words, the $X / R$ ratio in PCC is equal in the source and the fault side). Even though this seems to be a particular case, the objective is to analyze the performance of the algorithms with regard to the asymmetrical phase rotations, without considering the effects of phase jumps. The results of this analysis for dips type $C$ and $D$ are shown in Figs. 5. The second test consisted in the analysis of all the possible combinations of phase jumps and retained voltages, as previously described in Section V.1, but considering an asymmetrical phase rotation of $-20^{\circ}$, in order to understand the effects of the combination of both disturbances. Figs. 6 display the results of this second analysis for dips type $C$ and $D$.

Despite the fact that Fig. 4(a) shows that SCA algorithm is not sensitive to phase jumps, from Fig. 5(a), it can be noted that this algorithm is very sensitive to asymmetrical phase rotations. As a consequence, when the grid voltage is disturbed by a phase jump and a phase rotation, SCA algorithm fails, as shown in Fig. 6(a).

Basically, this behavior is explained by the variations that asymmetrical rotations introduce in the $\arg \left\{V_{-1} /(1-\right.$ $\left.\left.V_{+1}\right)\right\}$.

The analysis of SPA performance shows that the algorithm is very sensitive to both phase jumps and phase rotations (Figs. 4(b) and 5(b)). This behavior is partially evidenced in [13], and this error is attributed to the unbalance of the three-phase voltages, where the minimum voltage is actually the second minimum voltage. 
Finally, the performance of SVA algorithm is similar to that of SCA, that is to say, it is very sensitive to phase rotations (Fig. 5(c)). This behavior is explained by the inclination angle $\left(\varphi_{\text {inc }}\right)$ of the ellipse described by the rotation of the space vector, and giving rise to classification errors. Bands of approximately $30^{\circ}$ where SVA algorithm classifies the voltage events correctly can be noticed. These bands are the consequence of the $\pm 15^{\circ}$ tolerance bands described in Table II. Finally, Fig. 6(c) illustrates how the algorithm performance degrades notoriously when the phase jumps are combined with a constant asymmetrical phase rotation.

\section{V.3. Symmetrical Phase Rotations}

In order to verify the issues described in previous sections, the algorithms were evaluated with symmetrical phase rotations. Fig. 7(a) shows that SCA algorithm fails in almost all cases. On the other hand, SPA algorithm is not affected by symmetrical rotations because they do not change the absolute magnitudes of the six voltages employed (Fig. 7(b)). Finally, Fig 7(c) lists the results of
SVA algorithm, which evidence the classification errors when the rotation angle is greater than the $30^{\circ}$ bands derived from the algorithm thresholds.

\section{V.4. Pre-Fault Voltage Deviations}

Most references in the bibliography do not consider the case when the pre-fault voltage differs from the nominal or declared voltage (IEEE 1564-2014) and assume ideal operating conditions. To make a more comprehensive study, an evaluation of the classification errors produced by pre-fault voltage deviations with respect to the nominal voltage was carried out. The scenario was the particular case of a symmetrical prefault deviation, in which the three phases were equally affected.

Figures 8(a)-(c) show the results of SCA and SPA failure when the pre-fault voltage is lower than 0.967p.u. and 0.96p.u. in the case of SVA. These results were obtained for a balanced system; and errors increase if an asymmetrical condition is considered.

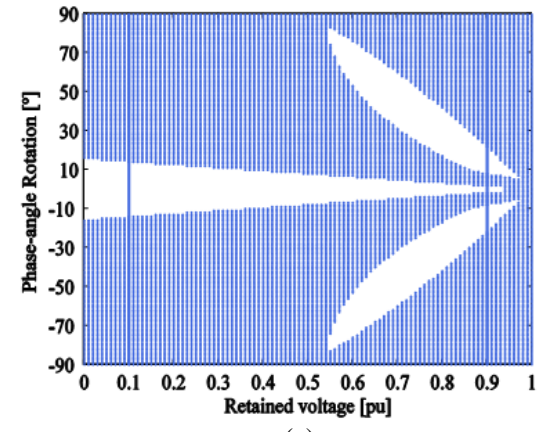

(a)

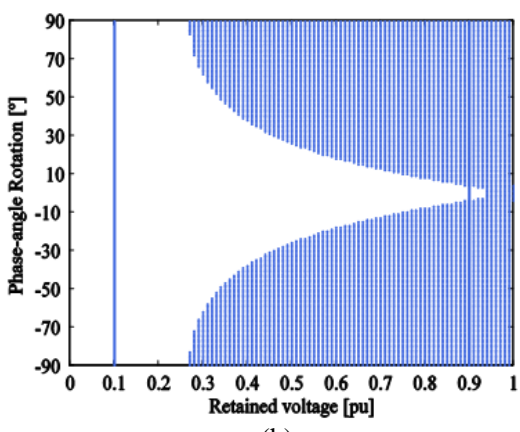

(b)

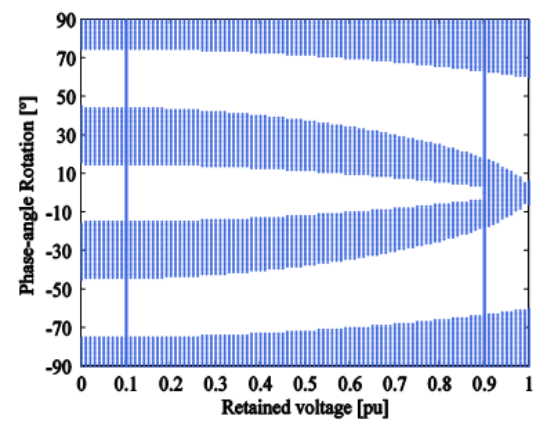

(c)

Figs. 5. Test of asymmetrical phase rotations of the SCA(a), SPA(b) and SVA(c) algorithms for voltage dips type C and D. Blue dots indicate that the classification performed by the algorithm was incorrect. Vertical lines for a retained voltage of 0.1 and 0.9 p.u. indicate the limits, within which the voltage dips are defined in the voltage dips according to IEEE 1159-2009

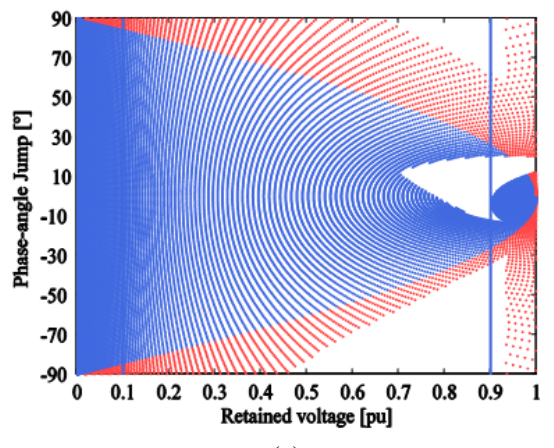

(a)

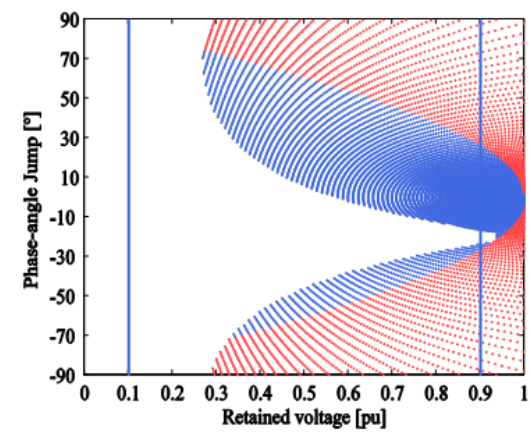

(b)

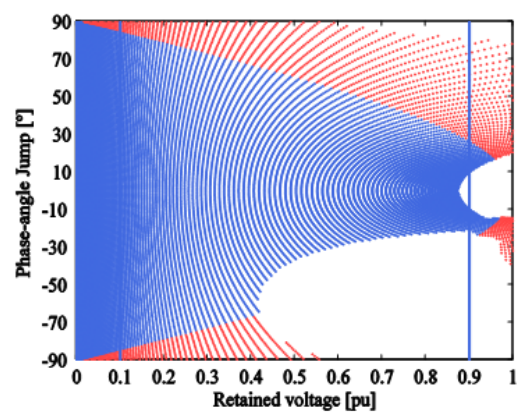

(c)

Figs. 6. Test of phase jump with an asymmetrical phase rotation of $-20^{\circ}$ of the SCA(a), SPA(b) and SVA(c) algorithms for voltage dips type C and D. Red and blue dots indicate that the classification performed by the algorithm was incorrect. Blue dots correspond to combinations of retained voltage and phase jumps that could occur in a radial network, while red dots correspond to combinations that should not be obtained in a radial network. Vertical lines for a retained voltage of 0.1 and 0.9 p.u. indicate the limits within which the voltage dips are defined according to IEEE 1159-2009 


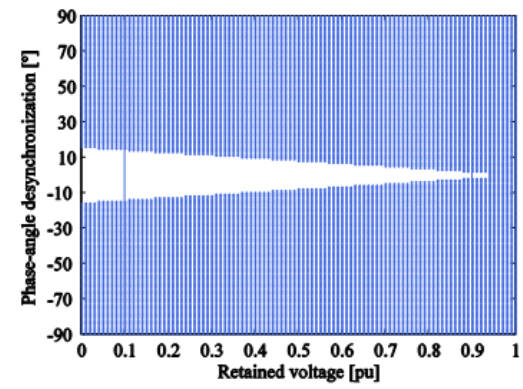

(a)

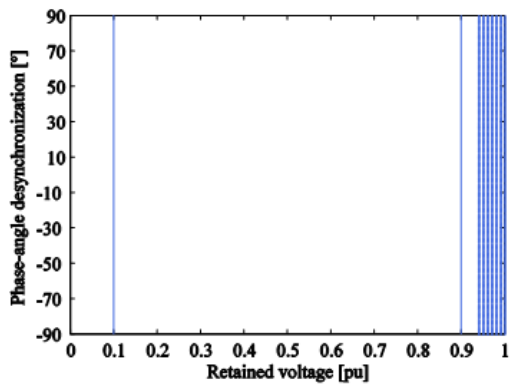

(b)

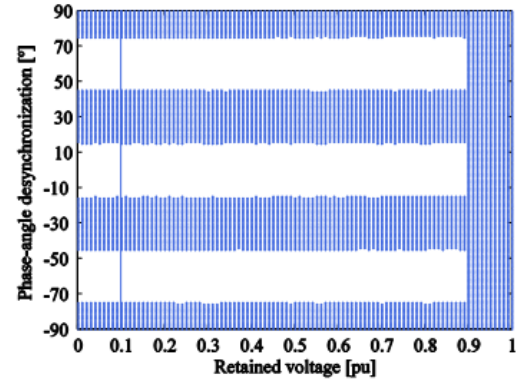

(c)

Figs. 7. Test of symmetrical phase rotations of SCA(a), SPA(b) and SVA(c) algorithms for voltage dips type C and D. Blue dots indicate that the classification performed by the algorithm was incorrect. Vertical lines for a retained voltage of 0.1 and 0.9 p.u. indicate the limits within which the voltage dips are defined according to IEEE 1159-2009

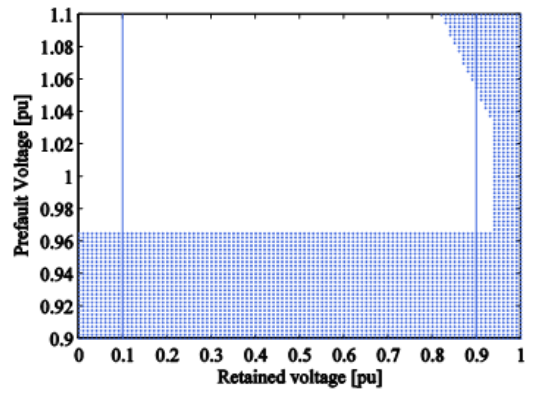

(a)

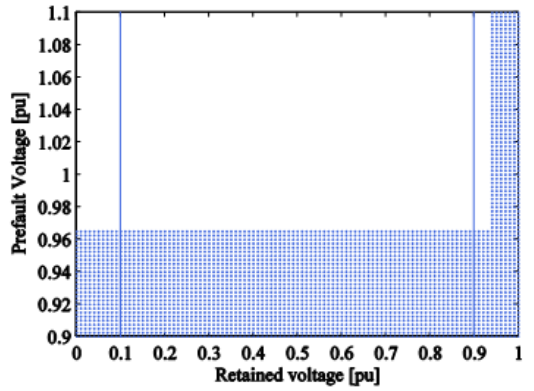

(b)

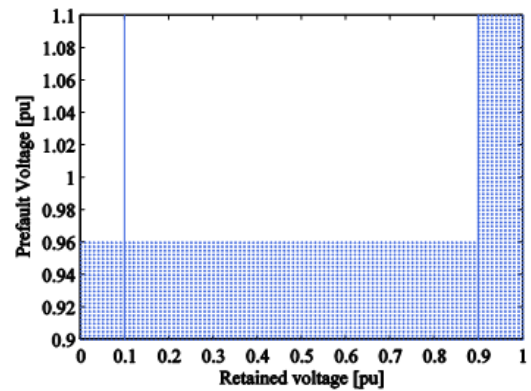

(c)

Figs. 8. Test of pre-fault voltage deviations of SCA(a), SPA(b) and SVA(c) algorithms for voltage dips type C and D. Blue dots indicate that the classification performed by the algorithm was incorrect. Vertical lines for a retained voltage of 0.1 and 0.9 p.u. indicate the limits within which the voltage dips are defined according to IEEE 1159-2009

\section{Discussion About the Causes of Algorithm Errors}

Below is a brief discussion of the errors in each specific algorithm. The aim is to understand the reasons why the chances of a univocal classification get reduced with these algorithms and criteria.

\section{VI.1. Symmetrical Components Algorithm}

Taking into account equation (2), the SCA classification is obtained from the positive and negative sequences components [13].

It fails in the presence of asymmetrical and symmetrical phase rotations because of the formulation of the algorithm itself and the ambiguity of the classification criterion when high phase rotations are considered. In the work by Bollen [13] the algorithm is modified, adding a constant phase angle of $20^{\circ}$ to the phase argument estimated with the sequence components (10). This modification mitigates the errors when the phase rotations (symmetrical and asymmetrical) are negative and of little magnitude. This partial solution does not include all the possible cases in actual implementations:

$$
T=\frac{1}{60}\left[\arg \left\{\frac{V_{-1}}{1-V_{+1}}\right\}+20^{\circ}\right]
$$

The positive and negative sequences should be known in order to understand the reasons why a misclassification occurs when equation (2) is applied.

Then, in this work, the transformation of symmetrical components is applied to the six equations that describe the voltages dips $C_{a}(1), C_{b}, C_{c}, D_{a}, D_{b}$, and $D_{c}$, obtaining (11):

$$
\left[\begin{array}{c}
V_{+1} \\
V_{-1}
\end{array}\right]=\left[\begin{array}{c}
\frac{F+V}{2} \\
\frac{F-V}{2} V_{\beta}
\end{array}\right]
$$

$V_{\beta}$ is a voltage phasor $\left|V_{\beta}\right| e^{j \beta}$ that represents a particular asymmetrical phase rotation for each event type. In other words, the positive sequences as a function of $F$ and $V$ parameters are independent of the event type, while in the case of the negative sequence there is a relation with the event type. From equation (11), it can be deduced that it is possible to characterize each event type with the phasor $V_{\beta}$, obtaining two solutions:

$$
\begin{gathered}
V_{\beta}=\frac{-V_{-1}}{V-V_{+1}} \\
V_{\beta}=\frac{V_{-1}}{F-V_{+1}}
\end{gathered}
$$


From (12) and (13), two new equations can be derived to estimate $T$ parameter and, consequently, classify the event without errors in the presence of phase rotations and jumps:

$$
\begin{aligned}
& T_{V}=\frac{1}{60^{\circ}} \arg \left\{\frac{-V_{-1}}{V-V_{+1}}\right\} \\
& T_{F}=\frac{1}{60^{\circ}} \arg \left\{\frac{V_{-1}}{F-V_{+1}}\right\}
\end{aligned}
$$

The dilemma is that $V$ and $F$ are variables estimated from $T_{V}$ and $T_{F}$, respectively, so, in order to calculate either of them, it is necessary to implement a recursive or iterative algorithm.

\section{VI.2. Six-Phase Algorithm}

This algorithm fails in the presence of big asymmetrical phase rotations and jumps. The reason of this classification error may be ascribed to the minimum RMS voltage that allows to determine the event type, which differs from the voltage prior to the phase jump.

A possible mitigation is to measure the first two minimum RMS voltages, estimate $F$ and $V$ values, and determine which of the possible events is more representative for the values of the characteristic magnitudes.

\section{VI.3. Space Vector Algorithm}

The performance analysis of the Space Vector Algorithm indicates that the classification errors occur in the presence of phase jumps associated to voltage dips of reduced magnitude, and phase rotations higher than $15^{\circ}$.

They originate in the deviation of the characteristic angle ( $\varphi_{i n c}$ or $\varphi_{01}-\varphi_{+1}$ according to the event type) with regard to the event type classification thresholds.

These errors may be partially solved by estimating the phase rotation or jump using parameters $V$ and $F$.

Nonetheless, as in the previous analysis, the ambiguity of the classification criteria prevents determining the event type without errors.

\section{VI.4. Pre-Fault Voltage Deviation}

The classification errors produced by the variations of the pre-fault voltage with regard to the nominal or declared voltage deserve special mention. In this case, it is necessary to work with variables that depend on the pre-fault voltage measurement and not on the nominal magnitudes, allowing an automatic and dynamic adjustment of the classification threshold.

An interesting approach is to estimate the pre-fault voltage in accordance with IEC 61000-4-30 Standard [21], where a sliding reference voltage is used.

\section{Conclusion}

This work presents an analysis of the drawbacks and constraints of voltage event classification criteria and algorithms. The analysis was performed based on two criteria and three different algorithms, whose performance was tested under the same operational conditions. The so-called Symmetrical Components and Six-Phase algorithms, originally proposed for SC criteria, were extended to contemplate $\mathrm{ABC}$ classification criteria, following suggestions from the literature; and a rigorous performance analysis of SVA, not available in the literature, was introduced.

It was demonstrated that the performance of the three algorithms is limited when voltage events are combined with phase rotations (symmetrical and asymmetrical), phase jumps, and/or pre-fault voltage deviations with regard to the nominal voltage. All these disturbances are common in actual networks and are directly related to the grid and load characteristics.

Despite the fact that some of these errors were reported in the literature for some of the algorithms, this work shows that these errors are not only produced by the algorithm architecture but also by the ambiguity of the classification criteria, which is not treated in the respective literature. In the case of SCA, (14) or (15) are more general and correct equations as compared to (2).

However, they depend on the event voltage type, so a recursive or iterative algorithm should be implemented. SPA and SVA algorithms can also be improved by estimating phase jumps or rotations.

Nonetheless, in the cases where the voltage event is combined with severe phase jumps or rotations, the classification becomes impractical. Based on these results, the need of a new classification criterion arises, using statistical information of actual faults, and more comprehensive description indices.

Finally, the importance of the voltage reference used in algorithms like SCA and SVA is underlined. Taking into account that both algorithms are designed for threephase voltages in relation to the pre-fault voltage, the cases in which the system is not based on this reference should be considered in order to obtain good performance in recursive implementations.

\section{Appendix}

\section{ABC Classification Equations}

Tables A1 and A2 exhibit the equations and diagrams corresponding to each dip and swell contemplated in $\mathrm{ABC}$ classification. In order to unify the criteria and to simplify the analytical study, all the equations are expressed with the same parameters of the Symmetrical Components criterion: retained voltage $\mathrm{V}$ and factor $\mathrm{F}$.

\section{Equivalent Model of the Symmetrical and Asymmetrical Faults}

The retained voltage in a voltage event of a radial 
network can be modeled with the single-line equivalent circuit of Fig. 1A.

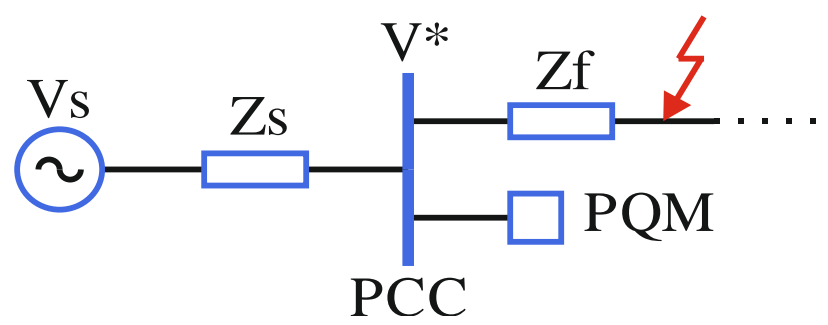

Fig. 1A. Simplified equivalent model for unbalanced and balanced three-phase voltage event

The retained voltage $V^{*}$ measured by a PQM (Power Quality Monitor) connected to the PCC (Point of Common Coupling) can be calculated from Eq. (1-A):

$$
V^{*}=\frac{Z_{f}}{Z_{f}+Z_{s}} V_{s}
$$

where:

- $V_{S}$ is the source voltage, which is the same as that of the pre-fault voltage when load current is negligible in comparison with the fault current.

- $Z_{f}$ is the impedance between PCC and the point where the fault is located.

- $Z_{S}$ is the impedance between PCC and the source.

Equation (1-A) is valid for three-phase faults, twophase faults and single phase faults, taking into account that in each case the values of $Z_{f}$ and $Z_{S}$ are calculated in a different way, as shown in Equations (2-A), (3-A) and (4-A).

Three-phase faults:

$$
\left[\begin{array}{c}
Z_{f} \\
Z_{s}
\end{array}\right]=\left[\begin{array}{l}
Z_{f+} \\
Z_{s+}
\end{array}\right]
$$

Two-phase faults:

$$
\left[\begin{array}{c}
Z_{f} \\
Z_{s}
\end{array}\right]=\left[\begin{array}{c}
Z_{f+}+Z_{f-} \\
Z_{s+}+Z_{s-}
\end{array}\right]
$$

Single-phase faults:

$$
\left[\begin{array}{c}
Z_{f} \\
Z_{s}
\end{array}\right]=\left[\begin{array}{c}
Z_{f+}+Z_{f-}+Z_{f 0} \\
Z_{s+}+Z_{s-}+Z_{s 0}
\end{array}\right]
$$

where $Z_{f+}$ and $Z_{S+}$ are the impedances of the positive sequence grid; $Z_{f-}$ and $Z_{S^{-}}$are the impedances of the negative sequence grid; while $Z_{f 0}$ and $Z_{S 0}$ are the impedances of the zero sequence grid.

Considering the impedance ratio $Z_{f} / Z_{S}=\lambda e^{j \alpha}$ in (1-A), the following expression is obtained:

$$
V^{*}=\frac{\lambda e^{j \alpha}}{\lambda e^{j \alpha}+1} V_{S}
$$

where $\lambda=|Z f / Z s|$ is a measurement of the electric distance to the fault and $\alpha=\arg (Z f / Z s)$ is a measurement of the maximum phase jump that could happen during a voltage event. This arises when analyzing that in expression (5A), if $\lambda$ tends to zero, $\left|V^{*}\right|$ tends to zero too, $\operatorname{and} \arg \left(V^{*}\right)$ tends to $\alpha$. Defining $h=\lambda e^{j \alpha} /\left(\lambda e^{j \alpha}+1\right)$, it can be observed that, when $\left(Z_{f} / Z_{S}\right)>>1$, or when the $X_{S} / R_{S}$ ratio is equal to the $X_{f} / R_{f}$ ratio, $\arg (h)=0$ and hence phase jumps do not appear in PCC. Generally, $\arg (h) \neq 0$ and so phase jumps appear in PCC, whose maximum value could reach $\alpha$, for instance, when $\left(Z_{f} / Z_{S}\right)<<1$.

TABLE A1

Voltage Dip Of The ABC Classification PROPOSED IN [1]

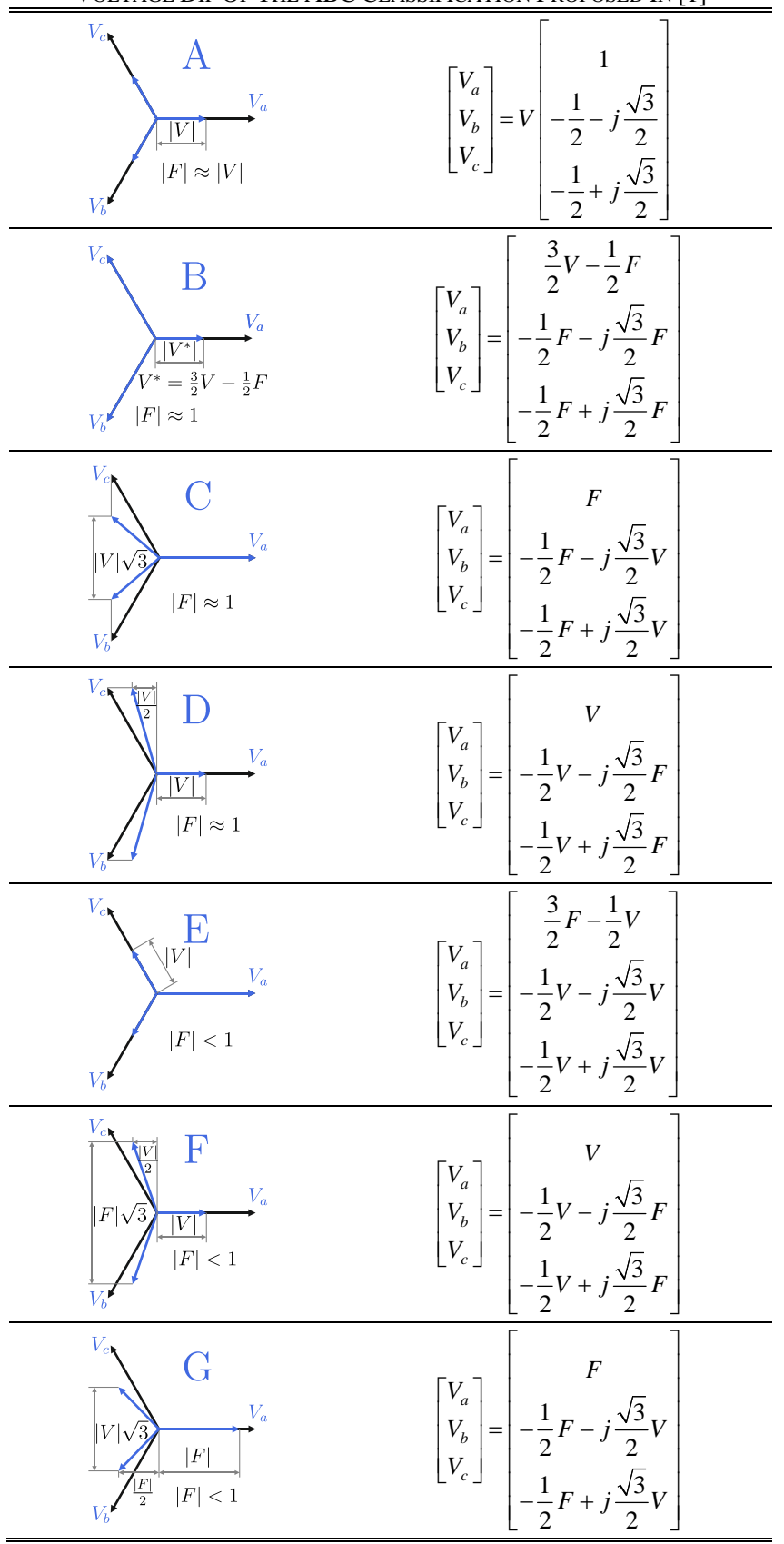


TABLE A2

VOLTAGE SWELl INCORPORATED TO THE ABC CLASSIFICATION IN [16]

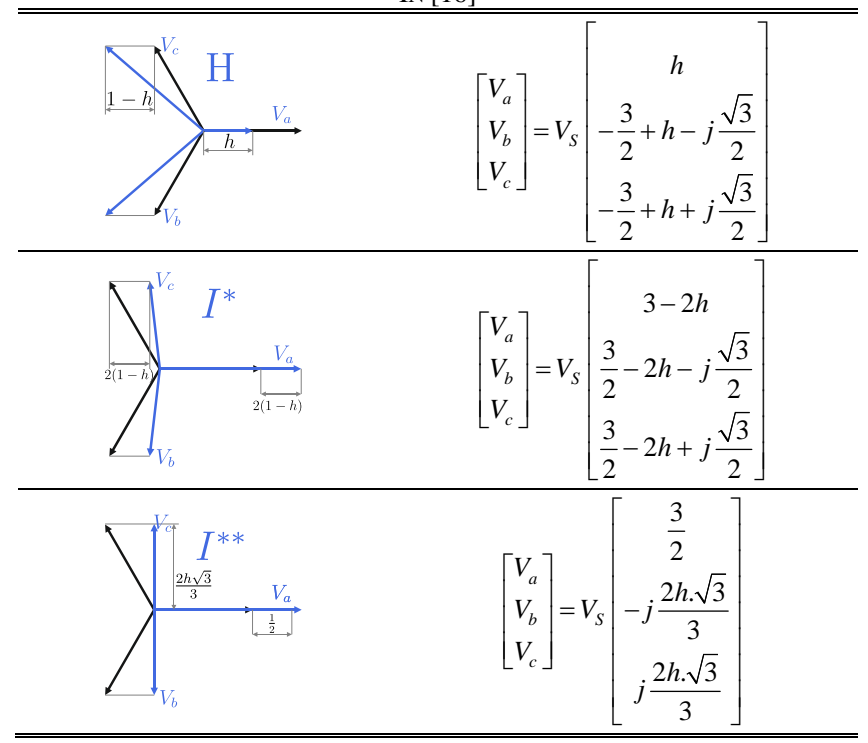

\section{Acknowledgements}

This work was supported by CONICET, CIC, Universidad Nacional de Mar del Plata, and ANPCyT.

\section{References}

[1] M. H. J. Bollen, Understanding Power Quality Problems: Voltage Sags and Interruptions, Wiley - IEEE Press, 1999.

[2] Standard, IEEE Std 1159 - IEEE Recommended Practice for Monitoring Electric Power Quality, 2009.

[3] L. L. Grigsby, Electric Power Generation, Transmission, and Distribution, 3rd Edition, CRC Press, 2012.

[4] A. dos Santos, M. C. de Barros, Stochastic modeling of power system faults, Electric Power Systems Research 126 (2015) 29 37. doi: http://dx.doi.org/10.1016/j.epsr.2015.04.015

[5] A. Bahmanyar, S. Jamali, A. Estebsari, E. Bompard, A comparison framework for distribution system outage and fault location methods, Electric Power Systems Research 145 (2017) $19-34$. doi: http://dx.doi.org/10.1016/j.epsr.2016.12.018

[6] O. Ipinnimo, S. Chowdhury, S. Chowdhury, J. Mitra, A review of voltage dip mitigation techniques with distributed generation in electricity networks, Electric Power Systems Research 103 (2013) $28-36$. doi: http://dx.doi.org/10.1016/j.epsr.2013.05.004

[7] M. H. J. Bollen, L. D. Zhang, Different methods for classification of three-phase unbalanced voltage dips due to faults, Electric Power Systems Research 66 (1) (2003) 59 - 69. doi: http://dx.doi.org/10.1016/S0378-7796(03)00072-5

[8] O. P. Mahela, A. G. Shaik, N. Gupta, A critical review of detection and classification of power quality events, Renewable and Sustainable Energy Reviews 41 (2015) 495 - 505. doi: http://dx.doi.org/10.1016/j.rser.2014.08.070

[9] S. Khokhar, A. A. B. M. Zin, A. S. B. Mokhtar, M. Pesaran, A comprehensive overview on signal processing and artificial intelligence techniques applications in classification of power quality disturbances, Renewable and Sustainable Energy Reviews 51 (2015) $1650-1663$. doi: http://dx.doi.org/10.1016/j.rser.2015.07.068

[10] M. K. Saini, R. Kapoor, Classification of power quality events - a review, International Journal of Electrical Power and Energy Systems 43 (1) (2012) $11-19$ doi: http://dx.doi.org/10.1016/j.ijepes.2012.04.045
[11] D. Granados-Lieberman, R. j. Romero-Troncoso, R. a. OsornioRios, A. Garcia-Perez, E. Cabal-Yepez, Techniques and methodologies for power quality analysis and disturbances classification in power systems: a review, IET Generation, Transmission Distribution 5 (4) (2011) 519-529. doi: http://dx.doi.org/10.1049/iet-gtd.2010.0466

[12] K. V. D. Saxena, S. Singh, Power quality event classification: an overview and key issues, MultiCraft International Journal of Engineering, Science and Technology 2 (3) (2010) 186 - 199. doi: http://dx.doi.org/10.4314/ijest.v2i3.59190

[13] M. H. J. Bollen, Algorithms for characterizing measured threephase unbalanced voltage dips, IEEE Transactions on Power Delivery 18 (3) (2003) 937-944. doi: http://dx.doi.org/10.1109/TPWRD.2003.813879

[14] V. Ignatova, P. Granjon, S. Bacha, F. Dumas, Classification and characterization of three phase voltage dips by space vector methodology, in: Future Power Systems, 2005 International Conference on, (2005). doi: http://dx.doi.org/10.1109/FPS.2005.204283

[15] M. H. J. Bollen, I. Y.-H. Gu, Signal Processing of Power Quality Disturbances, Wiley - IEEE Press, 2006.

[16] V. Ignatova, Méthodes d'analyse de la qualité de l'énergie électrique. Application aux creux de tension et á la pollution harmonique, $\mathrm{Ph} . \mathrm{D}$. thesis, Universite Joseph Fourier.

URL https://tel.archives-ouvertes.fr/tel-00170713

[17] V. Ignatova, P. Granjon, S. Bacha, Space vector method for voltage dips and swells analysis, IEEE Transactions on Power Delivery 24 (4) (2009) 2054-2061. doi: http://dx.doi.org/10.1109/TPWRD.2009.2028787

[18] M. H. Chia, A. M. Khambadkone, Subcycle voltage dip classification using matrix pencil method with ellipse fitting algorithm, IEEE Transactions on Industry Applications 51 (2) (2015) 1660-1668. doi: http://dx.doi.org/10.1109/TIA.2014.2347455

[19] M. R. Alam, K. M. Muttaqi, A. Bouzerdoum, Characterizing voltage sags and swells using three-phase voltage ellipse parameters, IEEE Transactions on Industry Applications 51 (4) (2015) 2780-2790. doi: http://dx.doi.org/10.1109/TIA.2015.2397176

[20] J. Suma, M. K. Mishra, Instantaneous symmetrical component theory based algorithm for characterization of three phase distorted and unbalanced voltage sags, in: 2013 IEEE International Conference on Industrial Technology (ICIT), 2013, pp. $845-850$.

doi: http://dx.doi.org/10.1109/ICIT.2013.6505782

[21] Standard, IEC 61000-4-30 - Electromagnetic compatibility (EMC) - Part 4-30: Testing and measurement techniques - Power quality measurement methods, 2015.

[22] Y. Wang, M. H. Bollen, A. Bagheri, X.-Y. Xiao, M. Olofsson, A quantitative comparison approach for different voltage dip characterization methods, Electric Power Systems Research 133 (2016) $182-190$. doi: http://dx.doi.org/10.1016/j.epsr.2015.12.032

[23] A. Sannino, M. H. J. Bollen, J. Svensson, Voltage tolerance testing of three-phase voltage source converters, IEEE Transactions on Power Delivery 20 (2) (2005) 1633-1639. doi: http://dx.doi.org/10.1109/TPWRD.2004.833881

[24] Standard, IEC 61000-4-11 - Electromagnetic compatibility (EMC) - Part 4-11: Testing and measurement techniques Voltage dips, short interruptions and voltage variations immunity tests, 2004.

[25] M. Bollen, P. Wang, N. Jenkins, Analysis and consequences of the phase jump associated with a voltage sag, in: $12^{\text {th }}$ Power Systems Computation Conference, 1996, pp. 316-322.

[26] M. H. J. Bollen, Characterisation of voltage sags experienced by three-phase adjustable-speed drives, IEEE Transactions on Power Delivery 12 (4) (1997) 1666-1671. doi: http://dx.doi.org/10.1109/61.634188

[27] S. Z. Djokic, J. V. Milanovic, Advanced voltage sag characterisation. Part I: Phase shift, IEE Proceedings Generation, Transmission and Distribution 153 (4) (2006) 423430. doi: http://dx.doi.org/10.1049/ip-gtd:20050350 


\section{Authors' information}

${ }^{1}$ Instituto de Investigaciones Científicas y Tecnológicas en Electrónica (ICYTE).

${ }^{2}$ Universidad Nacional de Mar del Plata (UNMdP), Juan B. Justo 4302, Mar del Plata, Argentina.

${ }^{3}$ Consejo Nacional de Investigaciones Científicas y Técnicas (CONICET), Av. Rivadavia 1917, Buenos Aires, Argentina.

E-mail: jlstrack@fi.mdp.edu.ar

Tel.: +54-223-481-6600 (int 254)

Fax: +54-223-481-0046

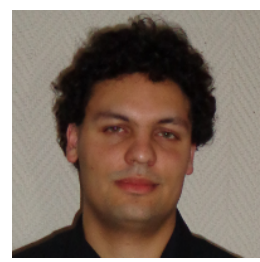

Jorge L. Strack was born in Argentina in 1989. $\mathrm{He}$ received the B.S. degrees in electromechanical engineering from Universidad Nacional de Mar del Plata, Mar del Plata, Argentina, in 2013, where he is currently pursuing the Ph.D. degree in electronics. He is currently with the Instituto de Investigaciones Científicas y Tecnológicas en Electrónica (ICYTE) of Universidad Nacional de Mar del Plata (UNMDP), Argentina; and is a member of the Consejo Nacional de Investigaciones Científicas y Técnicas (CONICET), Argentina. His current research interests include detection and classification of voltage events, power quality, digital signal processing and virtual instrumentation.

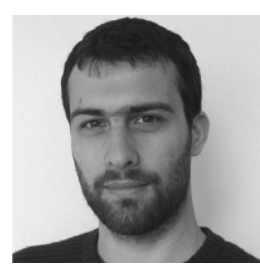

Ignacio Carugati was born in Argentina in 1983. He received the B.S. and Ph.D. degrees in electronics engineering from Universidad Nacional de Mar del Plata, Mar del Plata, Argentina, in 2008 and 2012, respectively. He is currently with the Instituto de Investigaciones Científicas y Tecnológicas en Electrónica (ICYTE) of Universidad Nacional de Mar del Plata (UNMDP), Argentina; and is a member of the Consejo Nacional de Investigaciones Científicas y Técnicas (CONICET), Argentina. His current research interests include control systems, power quality, smart grids and digital signal processing.

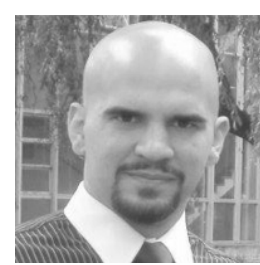

Carlos M. Orallo was born in Argentina in 1982. He was awarded his B.S. and Ph.D. degree in Electronics Engineering by the Universidad Nacional de Mar del Plata (UNMDP), Mar del Plata, Argentina, in 2011 and 2015 respectively. He is currently with the Instituto de Investigaciones Científicas y Tecnológicas en Electrónica (ICYTE) of Universidad Nacional de Mar del Plata (UNMDP), Argentina; and is a member of the Consejo Nacional de Investigaciones Científicas y Técnicas (CONICET), Argentina. His research interests include harmonics measurement, power quality, smart grids, and digital signal processing.

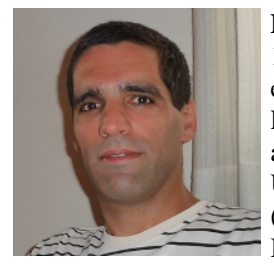

Patricio G. Donato was born in Argentina in 1975. He received the B.S. degree in electronics engineering from Universidad Nacional de la Patagonia San Juan Bosco (Argentina), in 2000, and the Ph.D. degree in electronics from Universidad de Alcalá, Alcalá de Henares (Spain), in 2005. He is currently with the Instituto de Investigaciones Científicas y Tecnológicas en Electrónica (ICYTE) of Universidad Nacional de Mar del Plata (UNMDP), Argentina; and is a member of the Consejo Nacional de Investigaciones Científicas y Técnicas (CONICET), Argentina. His current research interests include digital signal processing, power quality, and power line communications.

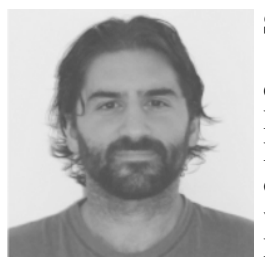

Sebastian Maestri was born in Argentina in 1978. He received the Electronics Engineering degree from the National University of Mar del Plata, Mar del Plata, Argentina, in 2005, and the $\mathrm{Ph} . \mathrm{D}$. degree in electronics from the University of Mar del Plata, Mar del Plata, in 2009. He was with the European Laboratory for Particle Physics, Centre Europeene pour la Recherche Nucleaire, Geneva, Switzerland, in control methods for improving the performance of thyristorized power supplies for bending-magnets. He is currently with the Instituto de Investigaciones Científicas y Tecnológicas en Electrónica (ICYTE) of Universidad Nacional de Mar del Plata (UNMDP), Argentina; and is a member of the Consejo Nacional de Investigaciones Científicas y Técnicas (CONICET), Argentina. His current research interests include power electronics, control systems and synchronism methods.

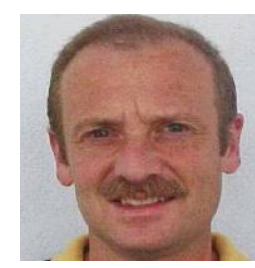

Daniel Carrica (M'84-SM'00) was born in Dolores, Argentine, in 1958. He received the Engineer and the PhD degrees from the National University of Mar del Plata (UNMDP), Argentine, in 1984 and 2006, respectively, and the MSc degree in Electronics from the Universidad Politécnica de Madrid, Spain, in 1992. In 1984 he joined the Department of Electronics (UNMDP), as a Research Assistant. From 1990 to 1999 he worked as Associate Scientific at CERN (European Organization for Nuclear Research), Geneva, Switzerland. From 1994 to 1996 he was Head of the Department of Electronics, UNMDP. He is currently Head of the ICYTE (Instituto de Investigaciones Científicas y Tecnológicas en Electrónica) of the UNMDP. Current research interests are Renewable Energies and Power Electronics. Dr. Carrica is Senior Member of IEEE and he was Chair of the Joint Chapter of Argentine IEEE Section during 2003-2004. 